\title{
Pseudomonas Aeruginosa as the Main Causative Agent of Osteomyelitis and its Susceptibility to Antibiotics
}

Author

Natalya N. Pliska

Affiliation

Research Institute of Traumatology and Orthopedics, Ministry of Health of the Republic of Kazakhstan, Nur-Sultan, Kazakhstan

Keywords

diagnostics, molecular pharmacology, pharmaceutics

received $\quad 03.03 .2020$

accepted $\quad 30.03 .2020$

Bibliography

DOI https://doi.org/10.1055/a-1150-2372

Published online: 17.4 .2020

Drug Res 2020; 70: 280-285

(c) Georg Thieme Verlag KG Stuttgart · New York

ISSN 2194-9379

\section{Correspondence}

Natalya N. Pliska

Research Institute of Traumatology and Orthopedics, Ministry of Health of the Republic of Kazakhstan

15A Abylay Khan Ave.

010000 Nur-Sultan

Kazakhstan

Tel.: 87025338891 , Fax : 87025338891

pliska5324@uoel.uk

\section{ABSTRACT}

Surgical activity is increasing in the treatment of many types of fractures, the use of various metal structures, and the potential for infection with the development of osteomyelitis accordingly increases. The urgency of the problem is due to the fact that this disease is the most expensive medical problem, especially when it comes to prosthetics of large joints, with socially significant losses and the occurrence of disability in patients of working age, it requires long-term treatment. The aim of this study was to study one of the most complex pathogens of life-threatening infections due to its high virulence and ability to adapt to changing environmental conditions, in particular the action of antibacterial drugs and the study of its sensitivity to certain groups of antimicrobial drugs. The results of the study showed that over the three years of observation in 2017-2019, the average value of Pseudomonas aeruginosa in the amount of $10.8 \%$ was established in the etiology of osteomyelitis. The revealed sensitivity of Pseudomonas aeruginosa is preserved to fluoroquinolone - levofloxanin, aminoglycosides-amikacin, gentamicin, carbapenems - meropenem, doripenem. All of the above drugs can be used as empirical therapy. During this period, a significant decrease in sensitivity was found, which reached in 2019 for cefepime $-51.9 \%$, pefloxacin $-55.8 \%$, ertapenem $-59.7 \%$. The success of the treatment of this pathology directly depends on the timely microbiological diagnosis and the choice of patient treatment tactics with the appointment of effective antibacterial therapy, with an adequate exposure of antibiotic.

\section{Introduction}

Constant development and improvements in the treatment of musculoskeletal injuries and long bone fractures have produced many methods of surgical treatment. However, an increasing surgical activity in the treatment of many types of fractures and the use of various metal structures increased the number of potential infections, which reached $5.9-13.6 \%$, which is a major and most expensive medical problem, especially when it comes to prosthetics of large joints, with socially significant losses and disabilities, up to $45 \%$ of cases in patients of active working age, $90 \%$ of which require a long-term treatment. Generalization of osteomyelitis can result in sepsis and death in some cases, which reach $1.2 \%$ [1-3]. In the UK, \$30-50 thousand is spent on the treatment of one patient with this pathology. In the United States, by 2020, the cost of treatment of osteomyelitis and other complications in traumatology and orthopedics is up to $\$ 1.620$ billion $[4,5]$.

The best results in the treatment of infections in the prosthetic joint or after an injury are achieved using a combination of a surgical intervention with a long-term etiotropic therapy [6]. As for the properties, an ideal antibacterial drug to treat an infection that occurs after an injury should be active against the pathogen in a particular patient, should have a bactericidal type of action, create high concentrations in bones, joints and soft tissues after parenteral or oral administration and have a favorable safety profile. Unfortunately, at present most antibiotics do not have either evidence base or a generally available experimental experience of their use in osteomyelitis [7]. The urgency of the problem of the treatment of chronic osteomyelitis is increasing due to a decrease in the effi- 
cacy of antibiotic therapy, prevalence of resistant strains of microorganisms and immune suppression due to unreasonable use of drugs [8].

The aim of our study was to study the isolated strains of Pseudomonas aeruginosa that caused chronic osteomyelitis in adults and to determine its susceptibility to antibiotics in order to increase the efficacy of treatment.

\section{Theoretical Overview}

In the structure of pathogens in traumatology and orthopedics, Pseudomonas aeruginosa, which causes osteomyelitis, is one of the most complex pathogens of life-threatening infections due to its high virulence and ability to adapt to changing environmental conditions, in particular the action of antibacterial drugs. The microorganism is quite insidious and aggressive, as evidenced by the development of infectious processes of various severity. Pseudomonas aeruginosa is highly common among the population as it is part of normal microflora (skin of the groin, underarm area, ears, nose, pharynx, gastrointestinal tract). Pseudomonas aeruginosa is ubiquitous; water plays an important part in the circulation of the pathogen, where it can survive up to one year (at $37^{\circ} \mathrm{C}$ ), including many solutions used in medicine. Up to $20 \%$ of all in-hospital or nasocomial infections are caused precisely by Pseudomonas aeruginosa, including $25 \%$ of purulent surgical processes. A quarter of the cases of primary bacteremia are also caused by Pseudomonas aeruginosa, an association of Staphylococcus aureus and Pseudomonas aeruginosa $(20.8 \%)[9,10]$.

Microorganisms of Pseudomonas species belong to the group of non-fermenting Gram-negative bacteria. They are free-living bacteria, extremely common in the environment, using almost all natural organic compounds as an energy source. The invasiveness of Pseudomonas aeruginosa is mediated by virulence factors; it produces an endotoxin, as well as a number of exotoxins. Exotoxin A causes local necrosis and facilitates the dissemination of the infection. The bacterium has the ability to withstand the action of resistance factors, which causes great problems for the etiotropic treatment $[11,12]$. In hospitals, highly resistant to antibiotics and antiseptics strains are common. The infection can occur both exogenously and endogenously. Various risk factors and concomitant diseases, such as rheumatoid arthritis, post-trauma surgery, obesity, coagulation disorders, preoperative anemia, diabetes mellitus and existing immunosuppression increase the possibility of osteomyelitis [13, 14].

Due to the ability to live in a humid environment, pseudomonads contaminate household items: medical instruments and equipment, medicines, a wide variety of disinfectant solutions, as well as, especially in places where liquids accumulate, in sinks, taps, dishes, respirators, humidifiers, dehumidifiers, air conditioners. As a consequence of the presence of pseudomonads in the environment of medical institutions, they cause colonization of the mucous membranes and skin of the patients. Pseudomonas aeruginosa has the ability of nonspecific adhesion to implantable devices (catheters, endotracheal tubes, etc.). There is also a mechanism of specific adhesion: the molecules that make up plasma proteins are adhesins for microorganisms. Adhesion increases with impaired mucociliary transport, which develops in the vast majority of intensive care unit (ICU) patients, in postoperative or post-traumatic periods, with acute heart and respiratory failure, any dehydration, and in all cases of mechanical ventilation. Subsequently, the microcolonies of bacteria form a continuous biofilm, which consists of several layers of microbial cells coated with common glycocalyx (a polysaccharide polymer). The vast majority of the cells are at rest and are characterized by extremely low sensitivity to the action of antibiotics.

A direct cause of osteomyelitis is the penetration of pathogenic bacteria into the bone with the development of a purulent inflammatory process, due to an intrabone invasion by Pseudomonas aeruginosa. The triggering factors for the development of osteomyelitis include trauma, burns, frostbite, an acute reaction to stress of injuries and operations, etc. The most problematic for the selection of effective antibacterial therapy (ABT) for the treatment of osteomyelitis are patients in the ICUs of some surgical hospitals reaching $88.9 \%$. A prevalence of antibiotic-resistant strains of microorganisms occurs as a result of a decrease in the immune resistance of a macroorganism during its long-term treatment with antimicrobial drugs, as well as due to mistakes made during treatment. Most often, it is a wrong duration of use, which reaches $35 \%[15,16]$.

Despite the presence of a large number of virulence factors, infections are rarely observed in individuals with normal resistance and intact anatomical barriers. The main role in the pathogenesis is played by the toxins of the pathogen. Most strains have surface villi that provide adhesion to the epithelium and the onset of the infectious process. Interactions with the cells are realized through receptors and a certain role is played by the produced mucus. It is a typical extracellular parasite, reproduction of which directly depends on the ability to withstand the action of resistance factors [17]. The best results in the treatment of infections in a prosthetic joint or after an injury are achieved using a combination of a surgical intervention with a long-term etiotropic therapy $[8,18]$. As for the properties, an ideal antibacterial drug to treat an infection that occurs after an injury should be active against the pathogen in a particular patient, should have a bactericidal type of action, create high concentrations in bones, joints and soft tissues after parenteral or oral administration and have a favorable safety profile. Unfortunately, at present most antibiotics do not have either evidence base or a generally available experimental experience of their use in osteomyelitis [19].

\section{Materials and Methods}

The study was conducted in compliance with the Helsinki Declaration of the World Medical Association on the ethical principles for medical research in humans adopted by the 59th General Assembly of the WMA Seoul, October 2008. Before hospitalization at the clinic, patients were unsuccessfully treated in other medical institutions for a long time. Various surgical interventions, from 3 to 5 , were performed in these patients. $64.2 \%$ of the patients underwent up to 3 interventions, $23.4 \%$ - from 4 to 5 interventions, $12.4 \%$ more than 5 interventions. Concomitant somatic pathologies were reported in 203 (81.5\%) of the examined patients, including cardiovascular, respiratory, genitourinary, endocrine, gastrointestinal diseases, ENT pathologies or alcoholism. In addition to Pseudomonas aeruginosa, $76.5 \%$ of the examined patients had mixed infections, of which Staphylococcus aureus was found in every fourth case ( $25 \%)$. 
All patients underwent a comprehensive examination, including clinical, biochemical, radiological and other tests. A necessary component of the preoperative study were tests for microflora and antibiotic susceptibility tests in fistulous drainage or puncture from the focus of osteomyelitis. The object of the study included smears from the wound, fistulous canal, punctate, as well as collected biomaterial after surgery, in cases of repeated revision of the joint or its replacement. All patients admitted with chronic osteomyelitis at the RSE Scientific Research Institute of Traumatology and Orthopedics, Nur-Sultan during 2017-2019, who came from all regions of Kazakhstan, were studied.

The biomaterials were studied using conventional methods; it was subjected to quantitative bacteriological analysis. The primary cultures of the biomaterials were carried out on various nutrient media; Gram staining and identification of the isolated microorganisms was carried out using the classical bacteriological method for the study of morphological, cultural and biochemical properties [20]. Antibiotic susceptibility tests of the isolated strains were performed using the standard disc method according to clinical recommendations [21]. The data obtained was processed using variation statistics [22].

\section{Results and Discussion}

The results of the study of the etiology of osteomyelitis following traumatological and orthopedic care were as follows. In 2017, 966 biomaterial studies with this nosological form were carried out, 13 of which showed negative results, which is $1.3 \%$. In 2018, 967 studies were performed with 310 (32\%) negative results, and during 11 months of 2019, 1202 studies showed 426 negative results (35.4\%). In 2017, Pseudomonas aeruginosa was identified in 90 (9.4\%) cases, in 2018 in 82 (12.5\%) cases, in 2019 in 77 (10.5\%) cases of the total number of identified microflora. Over three years, 23 types of microorganisms were isolated in wound discharge, but the main role in postoperative complications was played by 4 main species: Staphylococcus aureus, Staphylococcus epidermidis, Pseudomonas aeruginosa, E. Coli, which are presented in $>$ Table 1.

Over three years, in the wound discharge of the patients suffering from osteomyelitis, according to our study, Pseudomonas aeruginosa occupied the third position with an average $10.8 \%$ of inoculations ( Table 1). The distribution of this pathogen in the wound discharge in the patients by year: in $2017-9.4 \%$, in $2018-12.5 \%$, in $2019-10.5 \%$. Our study showed a two-fold lower prevalence of Pseudomonas aeruginosa in the etiology of chronic osteomyelitis in adults compared to foreign and Russian authors [23-26].
Antibiotic susceptibility tests that were used in the laboratory showed a high level of susceptibility of Pseudomonas aeruginosa to most antimicrobials only in 2017. The lowest susceptibility was found in the group of $\beta$-lactam antibiotics, the mechanism of action of which is based on the bactericidal effect and the presence of a betalactam ring in the chemical structure which blocks the synthesis of the cellular wall elements. Susceptibility to this group of the drugs decreased over three years of the studies. Thus, susceptibility to cefepime, which belongs to the IV generation of cephalosporins, was identified only in $70.5 \pm 4.8 \%$ strains in 2017 , in $64.0 \pm 5.3 \%$ in 2018 and $51.9 \pm 5.7 \%$ in 2019 . A slightly higher sensitivity was observed to ceftazidime, a III generation cephalosporin, i.e. $90.0 \pm 3.2 \%$ in $2017,81.7 \pm 4.3 \%$ in $2018,76.6 \pm 4.8 \%$ in 2019 . Penicillins, antibiotics of the $\beta$-lactam group, protected from the action of a bacterial enzyme that blocks $\beta$-lactamase synthesis, were represented by timentin (which contains clavulonic acid) and its susceptibility decreased (by $9.3 \%$ ) over the study period, > Table 2 .

Fluoroquinolones are able to penetrate through the outer membrane of Gram-negative microorganisms (including pseudomonads), avoiding the porin channels. Lipophilic antibiotics penetrate fairly well through the cytoplasmic membrane into the cytoplasm, where their action targets, topoisomerase enzymes, are localized. Of this group of hydrophobic antibiotics, ciprofloxacin is the most clinically important, with the highest antiseptic activity. The group of fluoroquinolones in our studies showed higher susceptibility compared to the group of $\beta$-lactam antibiotics. $82.2 \pm 3.3 \%$ of strains were sensitive to ciprofloxacin in $2017,82.9 \pm 4.2 \%$ in 2018 , and $74.0 \pm 4.9 \%$ in 2019. Susceptibility to pefloxacin was the weakest in 2017 , $70.0 \pm 4.8 \%$ of the strains, in 2018 it was $71.9 \pm 4.9 \%$ and $55.8 \pm 5.7 \%$ in 2019 respectively. In this group of antibiotics, the highest susceptibility was demonstrated by levofloxacin, $90.9 \pm 3.03 \%$ in 2017 , $89.5 \pm 3.4 \%$ in 2018 and $80.5 \pm 4.5 \%$ in 2019 . A decrease in susceptibility was also observed in this group of antibiotics (ciprofloxacin by $10.2 \%$, pefloxacin by $16.1 \%$ and levofloxacin by $10.4 \%$ ), on average by $3.9 \%$ per year.

The next group of antibiotics studied regarding the susceptibility of Pseudomonas aeruginosa were aminoglycosides. The structural features of the outer membrane and the release system of Pseudomonas aeruginosa have low effects on the level of the natural activity of aminoglycoside antibiotics. The minimum inhibitory concentrations of aminoglycosides regarding Pseudomonas aeruginosa are close to those for other Gram-negative bacteria. The resistance of Pseudomonas aeruginosa to aminoglycoside antibiotics is formed via three mechanisms: modification of the ribosome binding site with antibiotics, decreased transport into the bacte-

- Table 1 Major pathogens of osteomyelitis during 2017-2019.

\begin{tabular}{|c|c|c|c|c|c|c|c|c|}
\hline \multirow[t]{2}{*}{ Microorganism } & \multicolumn{2}{|c|}{2017} & \multicolumn{2}{|c|}{2018} & \multicolumn{2}{|c|}{2019} & \multicolumn{2}{|c|}{ Total } \\
\hline & abs. & $\%$ & abs. & $\%$ & abs. & $\%$ & abs. & $\%$ \\
\hline Staphylococcus aureus & 426 & 44.7 & 332 & 50.5 & 310 & 42.5 & 1068 & 45.9 \\
\hline Staphylococcus spp. & 286 & 30.01 & 129 & 19.6 & 124 & 17.0 & 539 & 23.2 \\
\hline Pseudomonas aeruginosa & 90 & 9.4 & 82 & 12.5 & 77 & 10.5 & 249 & 10.7 \\
\hline Escherichia coli & 42 & 4.4 & 37 & 5.6 & 35 & 4.8 & 114 & 4.9 \\
\hline Other microorganisms & 109 & 11.49 & 77 & 11.8 & 170 & 23.3 & 356 & 15.3 \\
\hline Total & 953 & & 657 & & 730 & & 2326 & \\
\hline
\end{tabular}


> Table 2 Antibiotic susceptibility of Pseudomonas aeruginosa over 2017-2019.

\begin{tabular}{|c|c|c|c|c|c|c|}
\hline \multirow{2}{*}{$\begin{array}{l}\text { Year } \\
\text { Antibiotic }\end{array}$} & \multicolumn{2}{|c|}{2017} & \multicolumn{2}{|c|}{2018} & \multicolumn{2}{|c|}{2019} \\
\hline & abs & $\% M \pm m$ & abs & $\% M \pm m$ & abs & $\% M \pm m$ \\
\hline Timentin & 71 & $80.7 \pm 4.1$ & 57 & $75.0 \pm 4.8$ & 55 & $71.4 \pm 5.1$ \\
\hline Ceftazidime & 81 & $90.0 \pm 3.2$ & 67 & $81.7 \pm 4.3$ & 59 & $76.6 \pm 4.8$ \\
\hline Cefepime & 62 & $70.5 \pm 4.8$ & 48 & $64.0 \pm 5.3$ & 40 & $51.9 \pm 5.7$ \\
\hline Ciprofloxacin & 74 & $82.2 \pm 3.3$ & 68 & $82.9 \pm 4.2$ & 57 & $74.0 \pm 4.9$ \\
\hline Levofloxacin & 80 & $90.9 \pm 3.03$ & 68 & $89.5 \pm 3.4$ & 62 & $80.5 \pm 4.5$ \\
\hline Pefloxacin & 63 & $70.0 \pm 4.8$ & 59 & $71.9 \pm 4.9$ & 43 & $55.8 \pm 5.7$ \\
\hline Gentamicin & 87 & $96.6 \pm 1.9$ & 78 & $95.1 \pm 2.4$ & 73 & $94.8 \pm 2.5$ \\
\hline Amikacin & 89 & $98.8 \pm 1.1$ & 80 & $97.6 \pm 1.6$ & 74 & $96.1 \pm 2.2$ \\
\hline Ertapenem & 88 & $97.8 \pm 1.5$ & 64 & $78.0 \pm 4.6$ & 46 & $59.7 \pm 5.6$ \\
\hline Meropenem & 90 & $100 \pm 0$ & 80 & $97.6 \pm 1.6$ & 71 & $92.2 \pm 3.05$ \\
\hline Doripenem & 0 & & 0 & & 75 & $97.40 \pm 1.8$ \\
\hline Total & 90 & & 82 & & 77 & \\
\hline
\end{tabular}

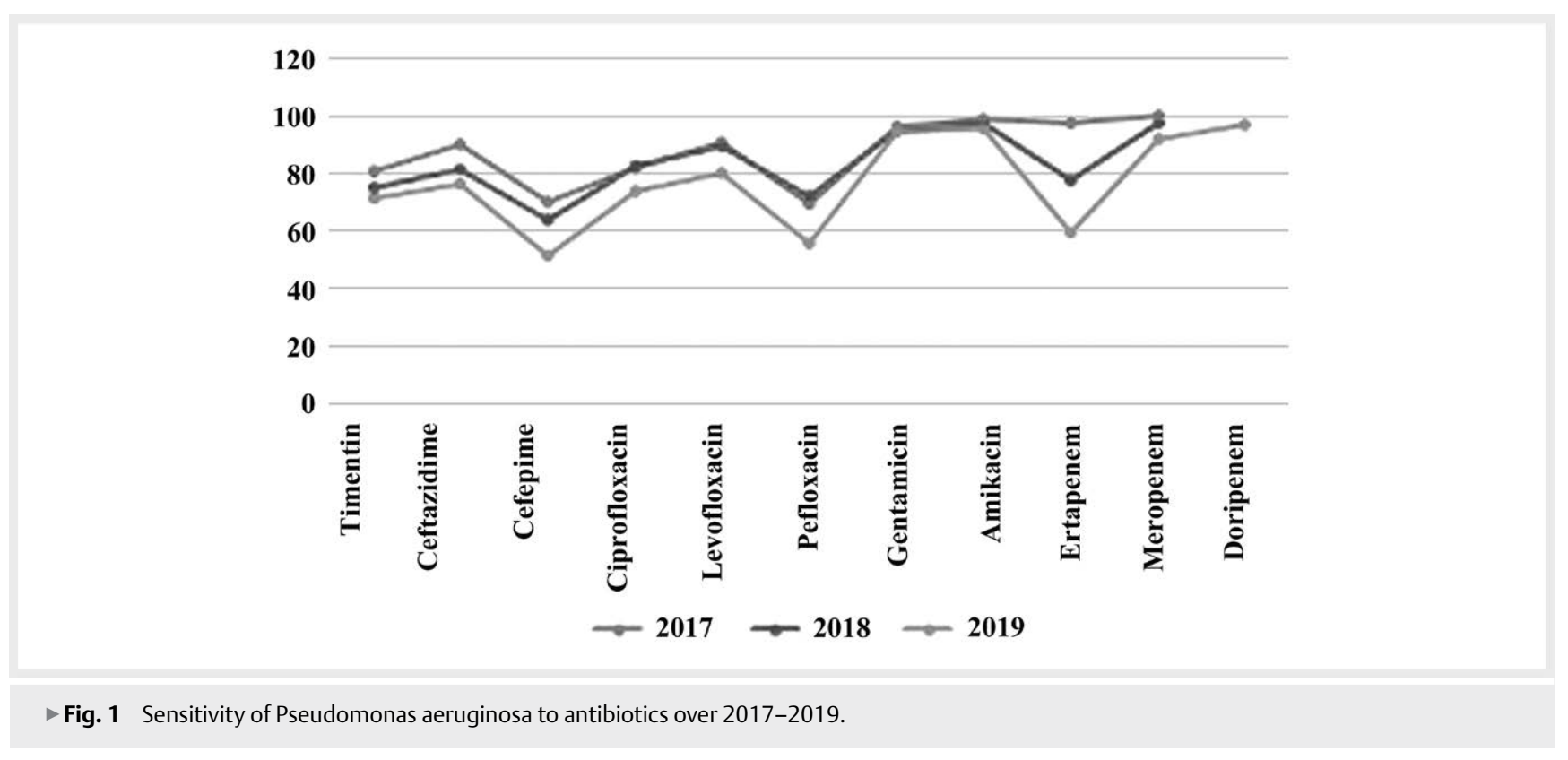

rial cell (impaired permeability of the inner or outer membrane), and enzymatic inactivation of antibiotics. Quite often, Pseudomonas aeruginosa strains can produce several enzymes simultaneously [1]. This group was studied on two representatives: gentamicin and amikacin. Susceptibility to gentamicin was observed in $96.6 \pm 1.9 \%$ of cases in $2017,95.1 \pm 2.4 \%$ in 2018 and $94.8 \pm 2.5 \%$ in 2019 . In $2017,98.8 \pm 1.1 \%$ of strains were sensitive to amikacin, in 2018 Pseudomonas aeruginosa was sensitive in $97.6 \pm 1.6 \%$ of cases, and in 2019 in $96.1 \pm 2.2 \%$ of cases. In this group of the drugs, a similar decrease in susceptibility was observed over the 3 studied years, in gentamicin by $0.6 \%$ and in amikacin by $0.9 \%$.

Carbapenems are leaders in the resistance to the action of lactamases. In our studies there were two representatives of this group: meropenem and ertapenem. Pseudomonas aeruginosa was $100 \%$ sensitive to meropenem in 2017 , the only high result in this strain, then it was observed a decrease in susceptibility by $2.4 \%$ and it reached $97.6 \pm 1.6 \%$ in 2018, in 2019 it decreased by $5.4 \%$ com- pared to 2018 and reached $92.2 \pm 3.05 \%$. Another representative of the group, ertapenem, lost susceptibility even more rapidly; in 2017 it was $97.8 \pm 1.5 \%$, in $2018-78.0 \pm 4.6 \%$ and in $2019-59.7 \pm$ $5.6 \%$; a decrease in susceptibility was by $38.1 \%$, which affected the addition of reserve antibiotics of this group and the introduction of doripenem in the algorithm in 2019. Pseudomonas aeruginosa showed high susceptibility to doripenem of $97.4 \pm 1.8 \%$.

The analysis of Pseudomonas aeruginosa susceptibility over three years is presented in $\mathbf{F i g . ~ 1 , ~ w h e r e ~ a u t h o r s ~ o b s e r v e ~ a ~ s i g - ~}$ nificant decrease in susceptibility to the following antimicrobial agents: ertapenem, pefloxacin, ciprofloxacin, cefepime and ceftazidime. To identify a high level of resistance of individual antibacterial drugs, a constant monitoring by microbiological laboratories is important in order to determine susceptibility of epidemiologically significant strains of microorganisms.

Therefore, monitoring changes in the sensitivity of Pseudomonas aeruginosa, isolated in patients with chronic osteomyeli- 
tis, over 2017-2019, it can be assumed that empirical treatment with III-IV generation cephalosporins is not possible, since their sensitivity decreases steadily. The use of this group of antibiotics is possible only after obtaining the result of a microbiological study.

Susceptibility to the group of fluoroquinolones varies; as for ciprofloxacin (74.0\% of the strains were sensitive in 2019), it cannot be used as an empirical therapy, unlike levofloxacin, to which susceptibility remains high - 80.5-95.5\%. Susceptibility of Pseudomonas aeruginosa to pefloxacin decreased significantly over three years of its monitoring (14.2\%) and in 2019 reached $55.8 \%$; such parameters indicate high resistance and the possibility of administration after obtaining the sensitivity result of the studied strain. Persistently high susceptibility to aminoglycosides (gentamicin and amikacin) was observed over two studied years, which supports their use in empirical therapy.

\section{Conclusions}

Susceptibility of Pseudomonas aeruginosa to antibacterial drugs is necessary to prescribe an adequate $A B T$ to patients with osteomyelitis, as well as to develop protocols for empirical antibiotic therapy. The strains were periodically susceptible to all antibiotics according to the guidelines on antibiotic sensitivity testing (EUCAST 2014). The isolates of Pseudomonas aeruginosa were most sensitive to doripenem ( $97.4 \pm 1.8 \%$ ), aminoglycosides (gentamicin and amikacin 94.8-96.1\%) and fluoroquinolones (ciprofloxacin and levofloxacin 74-80.5\%). The group of penicillins, which is represented by timentin, may be used as a treatment for Pseudomonas aeruginosa after obtaining the results of a microbiological study. Of all the studied antibacterial drugs, the lowest efficacy against Pseudomonas aeruginosa, especially in 2019, was shown by cefepime ( $51.9 \%$ of the strains were sensitive), pefloxacin ( $55.8 \%$ of the strains were sensitive) and ertapenem (59.7\% of the strains were sensitive).

Given the fact that $90-95 \%$ of patients come to our institute from all regions of Kazakhstan after 2-3 courses of unsuccessful $\mathrm{ABT}$, authors deal with a resistant microflora in the chronic process of osteomyelitis and the microorganism itself is highly contagious, therefore, it is necessary to consider treatment as a combination of adequate active and surgical tactics, carefully draining osteomyelitis foci surgically and using etiotropic antimicrobial therapy with two antibacterial drugs with good bioavailability for faster results and focus debridement, which will destroy the intrabone infection, reduce the rates of resistant strains of Pseudomonas aeruginosa, in the following combinations: 1) $\beta$-lactam antibiotics and aminoglycosides 2) $\beta$-lactam antibiotics and fluoroquinolones. Obviously, the development of uniform recommendations is impossible without local monitoring of the nature and level of antibiotic resistance.

Unfortunately, to date, there are no comparative randomized studies involving large groups of patients which analyze the efficacy of osteomyelitis treatment using different treatment algorithms. This is mainly due to the presence of many factors affecting the development and course of the infectious process. The patient's condition (concomitant pathology, bad habits, etc.), the nature of the pathogen (type), antibiotic resistance, equipment of the medical institution (equipment and tools for the operating room, labora- tory), as well as qualifications of the surgeon, clinical microbiologist, specialist in infectious diseases and clinical pharmacologist can significantly affect treatment outcomes.

In our opinion, the success of the treatment of this pathology directly depends on timely microbiological diagnosis, correct determination of its type and identification of the pathogen, which is crucial for the choice of treatment tactics for the patient and prescription of effective ABT. The treatment of this pathology is a complex task that requires a multidisciplinary approach and additional training for doctors involved in diagnosis and treatment.

\section{Conflict of interest}

The author declares no conflict of interest to disclose.

\section{References}

[1] Winkler T, Trumpusch A, Renz N. Classification and algorithm for the diagnosis and treatment of periprosthetic hip infection. Traumatology and Orthopedics of Russia 2016; 1: 33-45

[2] Tikhilov M, Shubnyakov II, Kovalenko AN. The structure of the early revisions of the hip joint replacement. Traumatology and Orthopedics of Russia 2014; 2: 5-13

[3] Bozhkova SA, Razorenov VL, Petrova TM. Microbiological monitoring is the basis of a rational strategy and tactics of antibiotic therapy for infection of bones and prosthetic joints. Togliatti Medical Council 2011; 3-4: 33-42

[4] Corvec S, Portillo ME, Pasticci BM et al. Epidemiology and new Developments in the diagnosis of prosthetic joint infection. International Journal of Artificial Organs 2012; 35: 923-934

[5] Zimmerli W, Trampuz A Biomaterials-associated infection: A perspective from the clinic. In: T.F. Moriarty, S.A.J. Zaat, H. Busscher, Eds., Biomaterials Associated Infection: Immunological Aspects and antimicrobial Strategies. New York: Springer; London: Heidelberg Dordrecht, 2013: 3-24

[6] Zimmerli W, Trampuz A, Ochsner P. Prosthetic-joint infections. New England Journal of Medicine 2004; 351: 1645-1654

[7] Geipel U. Pathogenic organisms in hip joint infections. International Journal of Medical Sciences 2009; 6: 234-240

[8] Ilyukevich GV, Smirnov VM, Levshina NN. Antibiotic resistance of hospital strains of Pseudomonas aeruginosa and optimization of the choice of antimicrobial therapy in intensive care and resuscitation departments. Infections and Antimicrobial Therapy 2003; 7: 63-67

[9] Rozova LV, Annuals NV. Comparative characteristics of the species composition of microorganisms in chronic post-traumatic and hematogenous osteomyelitis. Orthopedics Genius 2014; 2: 56-59

[10] The state of antibiotic resistance of gram-negative pathogens of nosocomial infections in the intensive care unit and intensive care unit, 1997. http://www.antibiotic.ru/iacmac/ru/pub/letters/argrmnoz/

[11] Rudnov VA. Antibiotic therapy for hospital infections caused by P. Aeruginosa. Russian Medical Journal 2005; 13: 485

[12] Harris AD, Perencevich E, Rohgmann M et al. Risk factors for piperacillin-tazobactam-resistant pseudomonas aeruginosa among hospitalized patients. Antimicrobial Agents and Chemotherapy 2002; 46: 854-858

[13] Bozic K], Lau E, Kurtz S et al. Patient-related risk factors for periprosthetic joint infection and postoperative mortality following total hip arthroplasty in edicare patients. Journal of Bone and Joint Surgery 2012; 94: 794-800 
[14] Perka C, Haas N. Periprothetische infektion. Der Chirurg 2011; 82 : 218-226

[15] Roy-Burman A, Savel RH, Racine S et al. Type III protein secretion is associated with death in lower respiratory and systemic pseudomonas aeruginosa infections. Journal of Infectious Diseases 2001; 183: 1767-1774

[16] Turner PJ. MYSTIC (Meropenem Yearly Susceptibility Test Information Collection): A global overview. Journal of Antimicrobial Chemotherapy 2000; 46: 9-23

[17] Pozdeev OK. Medical Microbiology. Moscow: Geotarmed; 2001

[18] Privolnev VV, Rodin AV, Karakulina EV. Topical use of antibiotics in the treatment of bone infections. Clinical Microbiology and Antimicrobial Chemotherapy 2012; 14: 118-131

[19] Winkler T, Trampush A, Renz N et al. Classification and algorithm for the diagnosis and treatment of periprosthetic hip infection. Traumatology and Orthopedics of Russia 2016; 1: 33-45

[20] Order of the Ministry of Health of the USSR of 04.22.1985. No. 535. On the unification of microbiological research methods used in clinical diagnostic laboratories of medical institutions. http://www.libussr.ru/ doc_ussr/usr_12667.htm
[21] Determination of the sensitivity of microorganisms to antimicrobial agents, 2014. http://www.antibiotic.ru/minzdrav/files/docs/ clrec-dsma2014.pdf

[22] Sergienko VI, Bondareva IB. Mathematical statistics in clinical studies. Moscow: GEOTAR Medicine; 2000

[23] Vincent J-L. Microbial resistance: lessons from the EPIC study. Intensive Care Medicine 2000; 26: S003-5008

[24] Trouillet JL, Chastre J, Vuagnant A et al. Ventilator-associated pneumonia caused by potentially drug-resistant bacteria. American Journal of Respiratory and Critical Care Medicine 1998; 157: 531-539

[25] Maksimkina EA, Vaskova LB, Musina NZ et al. Budget impact analysis of antipsychotic drugs in the hospital treatment of schizophrenia. Per Tche Quim 2019; 16: 509-515

[26] Dossa AB, Silva YPD. Anti-hypertension drugs: Action in the organism and environmental contamination. Per Tche Quim 2018; 15: 527-549 